\author{
Gregory A. Schmidt \\ Pierre Kory
}

\section{Ultrasound-guided central venous catheter insertion: teaching and learning}

Received: 26 August 2013

Accepted: 28 August 2013

Published online: 7 September 2013

(C) Springer-Verlag Berlin Heidelberg and ESICM 2013

This editorial refers to the article available at: doi:10.1007/s00134-013-3069-7.

\section{G. A. Schmidt (匹)}

Division of Pulmonary Diseases, Critical Care, and Occupational Medicine, University of Iowa, Iowa City, IA, USA

e-mail: gregory-a-schmidt@uiowa.edu

\section{P. Kory}

Pulmonary, Critical Care, and Sleep Medicine Division, Albert Einstein College of Medicine, New York, NY, USA e-mail: pkory@chpnet.org

It is now widely accepted that ultrasound-guided central venous catheter (CVC) insertion improves success, reduces complications, and represents the standard of care [1-6]. Where CVCs are used often, such as the intensive care unit, emergency room, and operating theater, trainees are schooled in procedural planning, ultrasound technique, and catheter insertion. Standardized approaches [7] and guidelines for training have recently been published [8]. But what is really being taught and learned? In this issue of Intensive Care Medicine, Nguyen and colleagues examined the pace at which trainees acquired knowledge and skills involved in jugular venous catheterization, finding that only 4-8 insertions were needed for competence [9]. Given the complexity of content, technique, and hand-eye-ultrasound coordination required, their results are encouraging and suggest that training is rapidly effective. This finding of a limited number of supervised procedures required to demonstrate competence is similar to skills in general intensive care ultrasound [10] and basic intensive care echocardiography [11, 12].

A decade ago, ultrasound-guided CVC insertion generally referred to internal jugular vein catheterization with the probe oriented transversely (showing a short-axis view of the vein) and the needle inserted along the longaxis of the vein. Regardless of clinical setting, speciality, or country of origin, most of us in a supervisory role were teaching very similar methods. This is no longer a safe assumption. The variety of techniques has exploded in the interim, involving which veins are catheterized (jugular, axillary, femoral, subclavian), where the probe is placed (showing the vein in either short- or long-axis), how the needle is oriented (in-axis with the vein, or out-of-axis, coming at a steep angle from above, right, or left [13]), and even where the operator stands (see Table 1). Some operators use needle-aiming devices, which have been reported to increase first-pass success for novices [14], whereas others find them cumbersome. The revelations of ultrasound are even fundamentally challenging how catheters are inserted, such as by calling into question the routine use of Trendelenburg positioning [15]. In a particularly novel report, clinicians dispensed with the syringe altogether when placing internal jugular catheters. Holding the needle directly and having the guidewire preloaded, they described enhanced manual dexterity; less risk of needle dislodgement (no syringe to remove); and superior needle visibility on ultrasound (due to the wire filling the lumen of the needle) [16]. Such a technique would simply not be possible without real-time imaging.

When judging what we teach and what our trainees learn, a detailed description of the procedural approach is essential. A strength of the article by Nguyen and colleagues is a clearly described method of insertion, further demonstrated in the accompanying video. The operator stands at the head of the patient, holds the transducer transversely to reveal a short-axis image of the vein, and punctures the vein from its lateral aspect (in line with the long-axis of the transducer, 
Table 1 Variety of techniques for ultrasound-guided CVC insertion

\begin{tabular}{|c|c|c|c|c|c|}
\hline Technique & Operator & Probe & Needle & Limitations & References \\
\hline Triangulation & Head & Short-axis & Long-axis & Tip not visualized & \\
\hline $\begin{array}{l}\text { Sliding or tilting } \\
\text { transverse }\end{array}$ & Head & Short-axis & Long-axis & & \\
\hline In-plane, long-axis & Head or left side & Long-axis & Long-axis & $\begin{array}{l}\text { Short neck can be challenging; } \\
\text { more skill required }\end{array}$ & [16] \\
\hline $\begin{array}{l}\text { In-plane, short-axis, } \\
\text { medial }\end{array}$ & Left side & Short-axis & $\begin{array}{l}\text { Short-axis, entering from } \\
\text { the left (medial) }\end{array}$ & $\begin{array}{l}\text { Needle must be re-oriented to } \\
\text { facilitate wire entry }\end{array}$ & [13] \\
\hline $\begin{array}{l}\text { In-plane, short-axis, } \\
\text { lateral }\end{array}$ & Head & Short-axis & $\begin{array}{l}\text { Short-axis, entering from } \\
\text { the right (lateral) }\end{array}$ & $\begin{array}{l}\text { Carotid risk; needle must be re- } \\
\text { oriented to facilitate wire entry }\end{array}$ & [9] \\
\hline
\end{tabular}

Orientation refers to insertion in the right internal jugular vein

allowing the needle tip and shaft to be visualized throughout its path towards and into the vein). Potential challenges of this method include keeping the needle precisely oriented within the narrow ultrasound beam (a limitation of all in-plane methods); risking carotid injury (because the artery, although medial to the vein, often remains within the path of the needle with this lateral approach); and reangling the needle to allow wire passage. Nevertheless, Nguyen's trainees performed this technique successfully, improved quickly, and had few complications. Yet this method is not widely used, so we must be cautious in generalizing these results. This caution is not unique to this study. In general, innovations in ultrasound-guided CVC insertion have spread without a corresponding proof of efficacy and safety. The attributes we associate with ultrasound guidance (success, complications, learning curve) may pertain to certain methods but not to all.

A second challenge in interpreting studies of the learning curve relates to specifically what is tested. For example, in the study of Nguyen, the individual components of the S1 and S2 scores are not intuitive and may impact the results. For example, why is "probe position at the lowest part of the neck" important and worth scoring? Including an arbitrary metric such as this, which is highly teachable, may bias the results by showing "improving" scores, not because the operator is getting better but because he is learning what the grader prefers. A similar criticism applies to S2. For example, most experts would not advocate routinely using color Doppler prior to CVC insertion in the internal jugular vein. Similarly, most operators will not say aloud "I've identified the vessel". If studied trainees learn that the study authors wish this, they will "improve" even if they have no heightened ability to visualize the needle tip or safely complete the procedure. In this regard, it is important that this study also showed rapid improvement in (arguably) more relevant measures such as time from skin puncture to venous flashback. Lastly, what is being tested may influence what is being taught. Given that adherence to sterile protocol in novice trainees is poor [17], the decision to focus on measuring the time for sterile preparation ("quantity") may negatively influence the number of violations of sterile protocol ("quality").

In the face of this complexity, there is general consensus around several key attributes for teaching safe ultrasound-guided CVC insertion. These should include (1) a curriculum clearly describing the technical approach and cognitive elements required, preferably with videobased procedural examples; (2) hands-on simulation training to develop hand-eye skills; (3) an emphasis on techniques that allow visualization of the needle tip at all times; and (4) insertions supervised by experienced clinicians giving feedback for improvement. The study by Nguyen shows that a modest investment in such a teaching approach produces real results for learning.

Conflicts of interest On behalf of all authors, the corresponding author states that there is no conflict of interest.

\section{References}

1. Lamperti M, Bodenham AR, Pittiruti M, Blaivas M, Augoustides JG, Elbarbary M, Pirotte T, Karakitsos D, LeDonne J, Doniger S, Scoppettuolo G, Feller-Kopman D, Schummer W, Biffi R, Desruennes E, Melniker LA Verghese ST (2012) International evidence-based recommendations on ultrasound-guided vascular access. Intensive Care Med 38:1105-1117
2. Prabhu MV, Juneja D, Gopal PB, Sathyanarayanan M, Subhramanyam S, Gandhe S, Nayak KS (2010) Ultrasound-guided femoral dialysis access placement: a single-center randomized trial. Clin J Am Soc Nephrol 5:235-239
3. Karakitsos D, Labropoulos N, De Groot E, Patrianakos AP, Kouraklis G, Poularas J, Samonis G, Tsoutsos DA, Konstadoulakis MM, Karabinis A (2006) Real-time ultrasound-guided catheterization of the internal jugular vein: a prospective comparison with the landmark technique in critical care patients. Critical Care 10:R162 
4. Fragou M, Gravvanis A, Dimitriou V, Papalois A, Kouraklis G, Karabinis A, Saranteas T, Poularas J, Papanikolaou J, Davlouros P, Labropoulos N, Karakitsos D (2011) Real-time ultrasound-guided subclavian vein cannulation versus the landmark method in critical care patients: a prospective randomized study. Crit Care Med 39:1607-1612

5. Randolph A, Cook D, Gonzales C, Pribble C (1996) Ultrasound guidance for placement of central venous catheters: a meta-analysis of the literature. Crit Care Med 24:2053-2058

6. ACS Committee on Perioperative Care (2011) Revised statement on recommendations for use of real-time ultrasound guidance for placement of central venous catheters. Bull Am Coll Surg 96:36-37

7. Feller-Kopman D (2007) Ultrasoundguided internal jugular access: a proposed standardized approach and implications for training and practice. Chest 132:302-309

8. Moureau N, Lamperti M, Kelly LJ, Dawson R, Elbarbary M, van Boxtel AJH, Pittiruti M (2013) Evidence-based consensus on the insertion of central venous access devices: definition of minimal requirements for training. $\mathrm{Br} \mathrm{J}$ Anaesth 110:347-356
9. Nguyen B-V, Prat G, Vincent J-L, Nowak E, Bizien N, Tonnelier J-M, Renault A, Ould-Ahmed M, Boles J-M, L'Her E (2013) Determination of the learning curve for ultrasound-guided jugular central venous catheter placement. Intensive Care Med. doi:10.1007/s00134-013-3069-7

10. Chalumeau-Lemoine L, Baudel JL, Das V, Arrivé L, Noblinski B, Guidet B, Offenstadt G, Maury E (2009) Results of short-term training of naïve physicians in focused general ultrasonography in an intensive care unit. Intensive Care Med 35:1767-1771

11. Melamed R, Sprenkle MD, Ulstad VK, Herzog CA, Leatherman JW (2009) Assessment of left ventricular function by intensivists using hand-held echocardiography. Chest 135:1416-1420

12. Vignon P, Mücke F, Bellec F, Marin B, Croce J, Brouqui T, Palobart C, Senges P, Truffy C, Wachmann A, Dugard A, Amiel JB (2011) Basic critical care echocardiography: validation of a curriculum dedicated to noncardiologist residents. Crit Care Med 39:636-642
13. Ho AM, Ricci CJ, Ng CS, Critchley LA, Ho AK, Karmakar MK, Cheung CW, Ng SK (2012) The medialtransverse approach for internal jugular vein catheterization: an example of lateral thinking. J Emerg Med 42:174-177

14. Augoustides JG, Horak J, Ochroch AE, Vernick WJ, Gambone AJ, Weiner J, Pinchasik D, Kowalchuk D, Savino JS, Jobes DR (2005) A randomized controlled clinical trial of real-time needle-guided ultrasound for internal jugular venous cannulation in a large university anesthesia department. J Cardiothorac Vasc Anesth 19:310-315

15. Nassar B, Deol GR, Ashby A, Collett N, Schmidt GA (2013) Trendelenburg position does not increase crosssectional area of the internal jugular vein predictably. Chest 144:177-182

16. Stone MB, Mallin M, Cook J (2013) Another WIN for point-of-care ultrasound: the wire-in-needle modified Seldinger technique for ultrasoundguided central venous access. Acad Emerg Med 20:E14-E15

17. Khouli H, Jahnes K, Shapiro J, Rose K, Mathew J, Gohil A, Han Q, Sotelo A, Jones J, Aqeel A, Eden E, Fried E (2011) Performance of medical residents in sterile techniques during central vein catheterization: randomized trial of efficacy of simulation-based training. Chest 139:80-87 